\title{
Integration of Traffic Management and Traveller Information Systems: Basic Principles and Case Study in Intermodal Transport System Management
}

\author{
Houcine Ezzedine, Therese Bonte, Christophe Kolski, Christian Tahon
}

\begin{abstract}
As they involve many interacting agents behaving in numerous ways that are extremely difficult to predict, urban transportation systems are complex in nature. The development of intermodal passenger transportation solutions to address the mobility issues constitutes a major thrust area of urban transport policies. But, to offer citizens comprehensive seamless mobility, intermodal transportation system management (ITSM) requires the integration of two major components. The traffic regulation support system, to help the operator responsible for the regulation tasks: coordination of timetables, synchronising arrival and departure times between the different transportation modes, and the traveller information system, giving customers access to information and using a comprehensive set of information tools. In this paper, a generic model of a transport management system, integrating these two components is proposed. This generic model is then used to elaborate a traffic regulation system in the case of a bimodal transportation system (tram-bus). The traffic regulation support system, based on the decision model of an operator, and the traveler information system are described.
\end{abstract}

Keywords: intermodal transportation systems, traffic regulation, travellers information system, management support system.

\section{Introduction}

Urban networks were, for years, only designed and built to carry flows of passengers to and from workplaces and their role was limited to their transport function. They did not take into consideration passenger expectations and the integration of the system in its environment. This period is definitely behind us, and a high transport capacity is no longer the sole criteria for assessing urban transportation system efficiency. Mobility patterns are hence changing as cities, with their large sprawling populations and are generating greater and more diverse mobility demands. Today, due to the growing service-oriented economy, people require a broad and flexible spread of transport services to choose from. Citizens are demanding greater mobility - more frequent and more widespread - and a higher quality mobility.

Most existing public transport systems were built at a time when the majority of journeys were fixed and routinely made. Therefore, they must now fundamentally change their approach. Public transport must take an increasingly flexible and competitive approach to meet the needs of today's travelling public and become more service-oriented. It must increase its quality, punctuality, frequency, attractiveness and comfort because all of which have an impact on its productivity. Indeed, urban networks have been transformed from just being a transport system into being an urban achievement around which the city and its mobility system are structured and developed. It is therefore crucial that an optimisation of interchange and transfer points between modes occurs for making them functional and pleasant. As well, there is no interest in setting up an efficient transport system if passengers do not know how to use it. So, another important aspect is the provision of high quality information. These two aspects, minimising waiting times and giving a real-time information to guide the travellers efficiently, are two essential points for the design of an intermodal transportation system.

The intermodal transportation systems approach is briefly described in the section II and a generic model of a traffic management system, integrating a traveller information system and a traffic regulation support system is proposed in the section III. The section IV shows how this model was used to develop a 
traffic regulation system, based on the decision model of an operator in the case of a bimodal transportation system (tram-bus). The travellers information system used by the operators is also explained. This system provides the operator with the information she/he needs to identify the disturbances and evaluate potential corrective actions to be carried out according to the regulation strategy she/he has selected. This paper is an extended variant of the work [15].

\section{Towards intermodal transportation systems}

Intermodality is both a technical term for a specific type of journey including several modes of transport and a policy principle. Intermodality describes coordinated interchanges between two or more transportation modes to complete a movement. In this light, intermodal movements involve either the physical transfer of people or individual items from one mode to another, or the transfer of one loaded transport vehicle or container from one mode to another to continue the journey. Numerous studies have been carried out on advantages (mainly quality of service) and disadvantages (costs, technical problems) of intermodal transportation systems. These are a number of barriers which prevent the advancement of intermodal passenger transportation (institutional, system integration, interoperability requirements) $[20,10]$. Nevertheless, the development of intermodal transportation solutions constitutes a main thrust area of urban transport policies and the role of intermodal transportation systems has become increasingly important. The intermodal approach involves looking at how individual modes can be connected and managed as a seamless and sustainable transportation system. That is, the fundamental objective of intermodalism is to integrate all the modes into an optimal, sustainable, and ethical system. Such a system should support efficiency, safety, mobility, economic growth, protection of the natural environment.

Any person departing from any of the available modes of transportation (train, bus, plane, metro, E) may get on the intermodal system additional services to reach its final destination. The comfort and the attractiveness of passenger transport systems depend largely upon the quality of the transfers at interchanges between public transport modes themselves and with the car. So, a crucial element of a successful intermodal transport system is the coordination of timetables, synchronising arrival and departure times between modes as much as possible to minimise passenger travel and waiting time. Here the impressive advances made in both computer technology, telecommunications and decision support tools and methods are playing a crucial role in the promotion of public transport: informing the travellers about existing transport possibilities in order to allow them to define and plan their movement, giving access to information and quality infrastructure to find out what mode of transport is available. In addition, parks and ride also contribute to a successful intermodal transport system, encouraging car drivers to leave their car in a car-park at the outskirts of the city and to travel by metro, tram or a bus on a route which is free of traffic difficulties.

The previous description shows that intermodal transport systems are customer-oriented that makes them really attractive. But, to offer citizens comprehensive seamless mobility, they require advanced management systems combining different decision and informational support, especially to help the operator, responsible for the traffic regulation.

\section{Generic model of a passenger traffic regulation support system}

The planning process of an urban transport system consists first in calculating a theoretical planning [1] and different timetables describe trips according to lines, frequencies, transport demand and travel times in the transport network. But, random events occur (vehicle's breakdown, strike, traffic congestion...) that cause disturbances and make the predictive scheduling to be modified. Therefore, for reducing the effects of disturbances, regulation tasks must be carried out to adapt the predictive scheduling to the real state of the network. This process consists in creating new schedules resulting from the 
decisions the regulator takes and depending on several parameters (the location, the type of disturbance, the time the disturbance occurs, ...).

The real-time regulation of an urban collective transport network is a very complex problem, especially in case of simultaneous disturbances, that frequently overload the regulator with numerous information. Taking efficient decisions requires: to have a global and significant overview of the network, to access rapidly to the available information related to the disturbances, to analyse the characteristics of the disturbances in order to identify the most appropriate corrective actions, to evaluate and compare the impact of these actions to decide which one must be selected. Our purpose here is to assist the regulators (decision-makers) and to propose them effective solutions taking account their preferences and uncertainties related to these preferences.

The generic model of a transport regulation support system (TRSS) is shown in figure 1 . The role of each software package as a component of the integrated architecture can be identified as follows:

- the Exploitation Assistance System is the main information system of the TRSS, interfacing TRSS with all the network information sources (sensors, GPS, phone, radio-locator...).

- the Decision Assistance System (DAS) is composed of three components: (i) the regulation data base containing all the information required for regulation (location of disturbances, line, bus

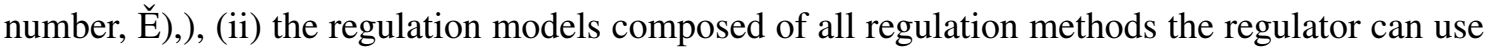
and/or combine to elaborate a coercive action: algorithms, meatheuristics,.. (iii) the RSS monitor which supports data manipulation, allows an interactive navigation through the regulation system, and allows the regulator to manage the regulation process by choosing the regulation models he wants to find out a solution.

- the Information Assistance System: it is used by the operators to send relevant information to the users of the network.

- the Geographical Information System (GIS): this module stores cartographic information about the network (streets and transportation networks, measurement locations, contour lines, etc.) and de-

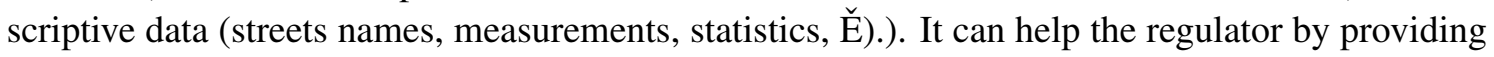
a spatial view of the network, locating and displaying the disturbances areas.

\section{Application}

The validation of our approach occurred within the framework of a project involving an industrial partner, the TRANSVILLES company, as well as several research laboratories (LAGIS, LAMIH and INRETS). TRANSVILLES is the company which actually runs the urban transport network (tramway and bus) in the town of Valenciennes. This project is sponsored by the Nord/Pas-de-Calais regional authorities and by the FEDER (Fonds Européen de Développement Régional - European Fund for Regional Development). Our research work consists in the specification, design and evaluation of (1) a humancomputer supervision interface (referred to later as the Information Assistance System or IAS), and (2) a Decision Assistance System (DAS). The IAS is intended for human regulators working on passenger information on the transport system in Valenciennes.

The transport network includes information screens (or display units) intended for the passengers. These screens are found both in the stations and in the vehicles; they show information on the schedules and on the connections. These information are calculated automatically by an exploitation assistance system (EAS) in which the position and state of each vehicle are stored.

In conclusion, the regulation system is made up of three sub-systems: an Information Assistance System (IAS), an Exploitation Assistance System (EAS), a Decision Assistance System (DAS), (see Figure 1). 


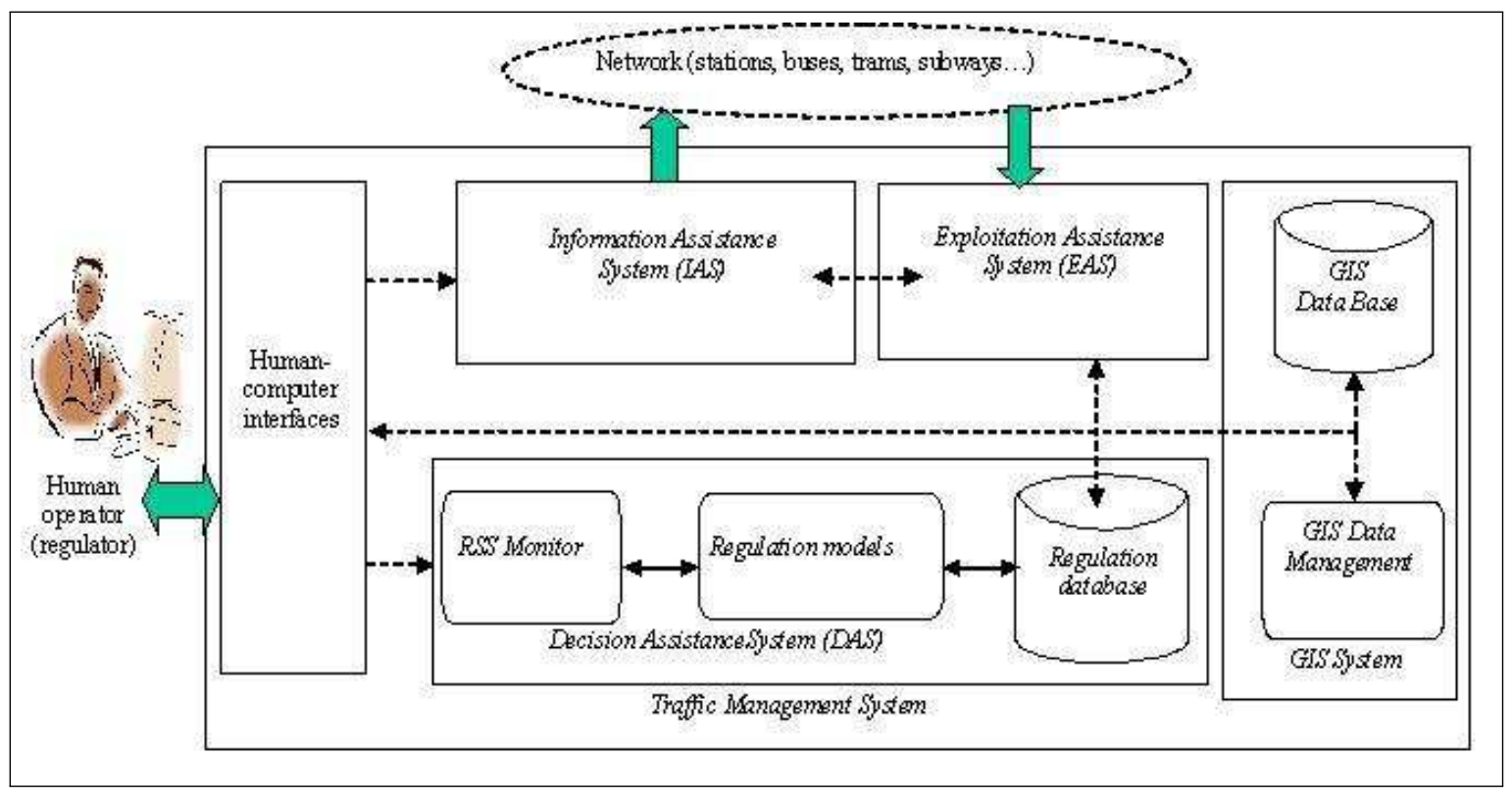

Figure 1: Generic model of an urban transport regulation support system

Each of the three sub-systems plays a particular role. Indeed, the EAS has to centralise the information regarding exploitation of vehicles (alerts, vehicles ahead of time, messages, schedules, delays,...), and make it possible to manage these elements. The DAS is intended to create, evaluate and suggest regulation strategies to the human regulator using the information provided by the EAS. In this way, the regulator has less work to do, which should help to improve the quality of regulation and thus the quality of the overall service. The DAS is not intended to replace the human regulator, but it must provide assistance in decision-making [8]. The IAS is intended to present information to the regulators and make it possible to send relevant information to the passengers [3, 4].

A simulation system has been developped to simulate the behaviour of the multimodal network in Valenciennes. This simulator is described in the next section. The DAS and the IAS, which are objects of our researches, are more detailed in the two next sections.

\section{The Simulator}

The software QUEST (Queuing Event Simulation Tool), developed by Delmia, was chosen to build the simulation model. QUEST is a powerful discrete event simulation tool that allows very complex systems to be modelled. We used it to model the transportation network of our industrial partner. This multimodal network is composed of buses, trams and trains. The simulation system takes both the normal behaviour and the disturbances into account. The topology of the transportation system consists in a road network principally used by buses and a rail network which incorporates both tram and train networks. This type of network is sometimes supported by a road network, in which case bus and tram interaction must be taken into consideration. Crossings are modelled to avoid collisions between buses or between buses and trams. Intersections between roads or between roads and rail lines are supervised. Trams always have priority in road-rail intersections. Overtaking is restricted: only stationary buses may be overtaken by moving buses. An overtaking manoeuvre on the rail network can only be accomplished by using a specific procedure, such as following parallel tracks or shunting tracks, which, strictly speaking, is traffic diversion, not overtaking. Rolling equipment can be divided into three categories: train, tram or bus. Buses can be further divided into standard buses, articulated buses or minibuses. Their dimensions, kinematic characteristics and capacities are modelled. Macroscopic passenger flow simulation is used to 
track passenger movements; the model does not track individual passengers. Passenger flow is considered at simple stop areas, at interchanges, and within the vehicles. Stop area arrival patterns are modelled using a Poisson law. The mean arrival rate per time unit varies according to timeband (period of the day) and type of day (weekday, weekend, school holidays Ě).). Stop areas are divided into simple stop areas and interchange stop areas. A stop area is simple if passengers can board or disembark without any possiblity of connecting to other lines while an interchange stop area combines multimodal transportation vehicles and may be very complex. In our application, the interchanges are divided into two types:

- interchanges between bus and tram: a tram drops passengers off, and when a bus reaches the stop area, it takes passengers on board and leaves, unless the bus driver sees an approaching tram. In this case, the bus waits for the tram. In any case, the tram drops its passengers off, regardless of whether there is an interchange or not,

- interchanges between bus and bus: the interchange is organized around an arriving bus, which brings passengers into the interchange, and a waiting bus, which takes waiting passengers out of the interchange.

Two types of disturbances may occur and disrupt the transportation network:

- alarms detected by the vehicle or its driver (failures, emergency calls) or detected by the controller (operational threshold exceeded $\check{\mathrm{E}}$ ))

- unforeseen events resulting from an accident, traffic jam, passenger behaviour, driver illness ... .

In our application, perturbations are generated by the user of the simulation system. Vehicle perturbations include delays, early arrivals, unscheduled stops, and network perturbations refers to the unavailability of some part of the network. The regulation action that will compensate for the dysfunction is defined by the user with the help of the Decision Assistance System and is then simulated. In this instance, QUEST's ability to communicate with external softwares via a socket-based mechanism that allows it to send or receive any type of information is clearly an advantage.

\section{The Decision Assistance System}

\subsection{Global modelling approach chosen concerning the decision-making process of the regulator}

DSS design is a very rich research and development field [21]. Most of the decision-making systems developed in the field of regulation are based on automatic methods. Several authors (see for instance [12]) have contributed to the use of artificial intelligence in the field of transportation system regulation using fuzzy models. Balbo [2] adopts a multi-agent approach for offer planning, and Laichour [8] uses the same type of approach to regulate transfers. Fayech Chaar [5] combines a multi-agent approach with a generic algorithm. However, none of these approaches consider the integration of the method in an interactive environment. In parallel, three main modelling approaches have been proposed and used to model a human operator:

- the oldest is the "human factor" approach (1940-1955), which models the observable elements of operator behavior during task execution [6].

- then, the "human/automatic" approach (1955-1980), which is based on physics theory, has tried to model the human operator. Information Theory, the Optimal Control Model and model of the regulation of human activity proposed by [9], are all based on this modelling approach. 
- the third approach is the "Cognitive engineering" approach, which holds that the role of human operators in system operations is supervision and decision-making. Thus, problems that this approach seeks to resolve are related to the decision-making involved in system control, management, monitoring and reconfiguration.

As we consider that the regulator is part of all supervision and decision-making tasks, our application falls into the "cognitive engineering" approach. Our proposed model is inspired by the ones introduced by Hoc [11] and [7] (see Figure 2). This model integrates the regulator in the decision process and allows conflicts between the user and the system to be avoided.

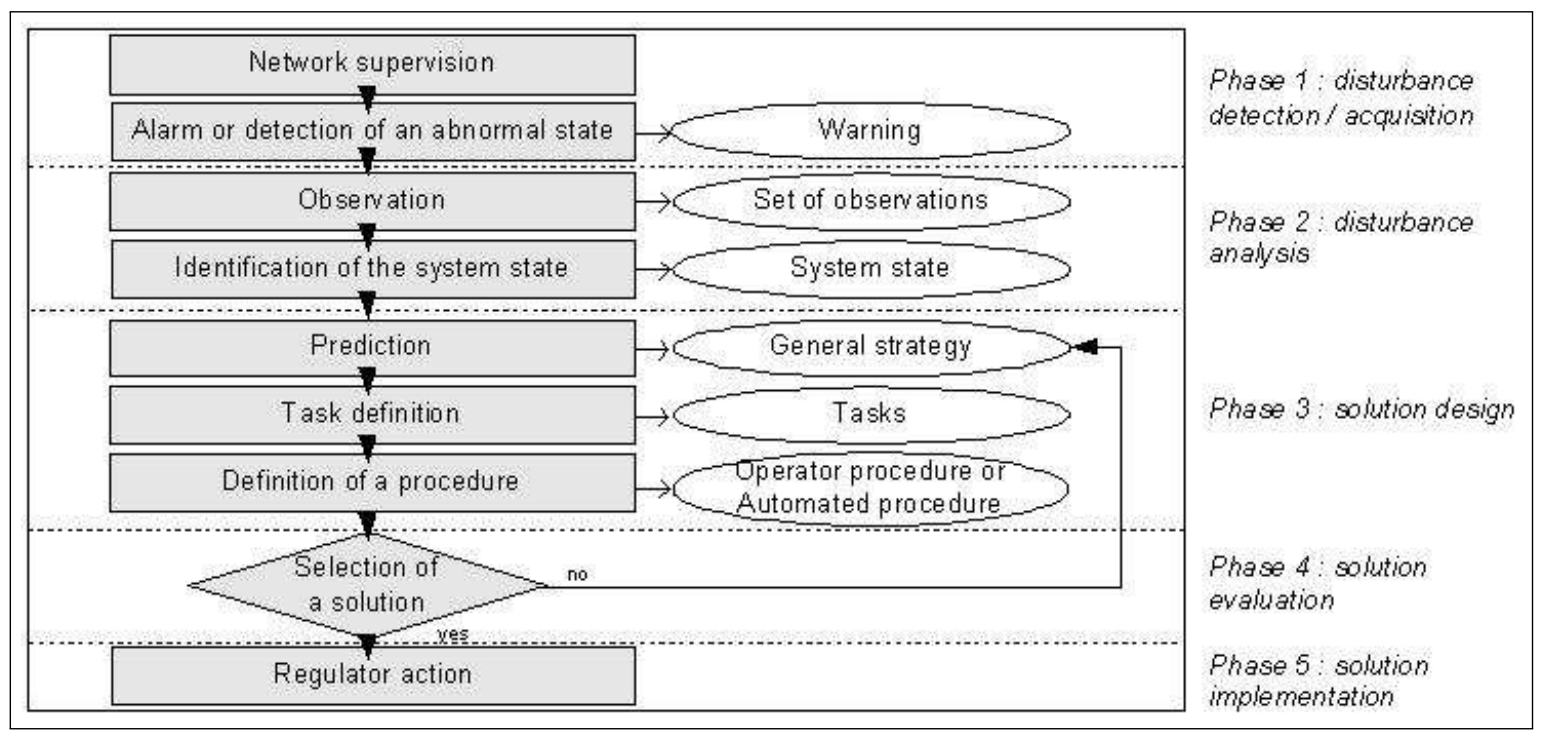

Figure 2: The decision model of the regulator

\subsection{Functional model for the regulation of a transportation system}

Figure 2 shows the functional diagram of the decision model described above. It is composed of five phases: disturbance detection and acquisition, disturbance analysis, solution design, solution evaluation and solution implementation. They are all described below.

- Phase 1: Disturbance detection/acquisition. Three detection methods are possible: (1) the automatic vehicle-monitoring system can provide relevant information about disturbances; (2) vehicle drivers can call in by radio; (3) the regulator can anticipate disturbances based on his experience in supervising a transportation network. The third case is the most common.

- Phase 2: Disturbance analysis. Descriptive data of a disturbance are numerous. Since there are often several simultaneous disturbances, the assistance system provides a disturbance analysis to avoid information overload at the level of the regulator. It provides a synthetic representation of relevant network data for each disturbance, a description of the specific information available (e.g., passenger flow at vehicle stops, number of passengers in the vehicles, characteristics of the line). Current disturbances are ranked according to significant criteria defined with the help of the industrial partner.

- Phase 3: Solution design. Three methods are proposed to the regulator for choosing or designing a solution. These methods complement approaches mentioned above [2] [5] [8]. They increase interactivity and allow the regulator to adapt to the varying complexity of the disturbances: 
- a manual method: all available basic and combined regulation actions are listed in a typology provided by the system and are proposed to the regulator [8].

- a semi-automatic method: the regulator chooses among the different regulation logics. He can focus on respect for punctuality or respect for transfers. The system can then filter acceptable actions and proposes a list of possible actions .

- an automatic method: the regulator chooses one of the suitable solutions automatically proposed by the system.

- Phase 4: Solution evaluation. All solutions are evaluated according to indicators computed by the system. These indicators allow the efficiency of the solution to be verified. These indicators are classified according to their importance: (1) "essential" indicators evaluate the service offered to passengers (e.g., regularity, punctuality, respect of transfer times); (2) "important" indicators evaluate the additional consequences of a disturbance (e.g., delays); (3) "secondary" indicators simply provide information to the regulator but have no influence on the decision (e.g., the lines and transfers that are affected by the solution). The regulator chooses the best solution according to these indicators.

- Phase 5: Solution implementation. The chosen solution is implemented by creating a new timetable.

\subsection{System components}

System implementation is based on an architecture including the following modules:

- The "acquisition tool" evaluates the complexity of the disturbances and classifies them based on local and network parameters and traffic management criteria (e.g., type of vehicle, geographical location, time, number of passengers in the vehicle and waiting at the next stop).

- The "regulation environment" manages three modules according to the method chosen by the regulator:

- the "manual tool module" is used if the regulator builds his own solution manually. This module contains a set of mathematical algorithms specifically developed for the project. Each algorithm models a specific regulation action such as "U-turn with vehicles and drivers exchange". These algorithms have been enhanced by a filtering procedure, which enables or disables the algorithms depending on which logic is chosen. The filter is based entirely on specifications defined by the industrial partner TRANSVILLES. For example "respect for transfer" logic will disable "U-turn with vehicles and drivers exchange".

- the "case-based reasoning module" uses the traditional CBR approach [19]. Its principle is based on the use of previous experience to solve problems. This approach can be used because TRANSVILLES has compiled a database of incidents and strategies applied to resolve them. This database is enriched with the activities resulting from manual regulation or those generated automatically by the system.

- the "expert system" uses a knowledge base that exploits the expertise of our industrial partner (TRANSVILLES). It is used if no solution can be found using the CBR module.

\section{The information assistance system}

\subsection{Architecture chosen for the human-computer interface}

Several architecture models of interactive systems have been put forward by researchers over the past twenty years. Two main types of architecture can be distinguished: architectures with functional 
components (Language, Seeheim and ARCH) and architectures with structural components (PAC, PACAmodeus, MVCĚ);); several of these models have also variations [13]. The classic models distinguish three essential functions (presentation, control and application). Some models (such as the Seeheim and ARCH models) consider these three functions as being distinct functional units. Other approaches using structural components, and in particular those said to be distributed or agent approaches, suggest grouping the three functions together into one unit, the agent. The agents are then organised in a hierarchical manner according to principles of composition or communication (for example PAC, MVC). Our approach could be considered as being intermediate as it borrows elements for its principles from both types of model given above: we suggest using a division into three functional components which we have called respectively: interface with the application (connected to the application), dialogue controller, and presentation (this component is directly linked to the user). These three components group together agents [14].

The application agents handle the field concepts and cannot be directly accessed by the user; one of their roles is to ensure the correct functioning of the application and the real time dispatch of the information necessary for the other agents to perform their task. The interactive agents (or interface agents) are in direct contact with the user; they co-ordinate between themselves in order to intercept the user commands and to form a presentation which allows the user to gain an overall understanding of the current state of the application. In this way, a window may be considered as being an interactive agent in its own right; its specification describes its presentation and the services it is to perform. The dialogue control agents provide services for both the application and the user; they are intended to guarantee coherency in the exchanges emanating from the application towards the user, and vice versa.

\subsection{Agent oriented specification and design of the human-machine interface}

An agent oriented architecture is used for the human-computer interface. The application agents are intended to manage the passenger information in the vehicles and stations and to calculate the information to be displayed (delays, timetable and route modifications, etc.). According to the traffic context, each agent possesses rules enabling it to act correctly in its environment. Concerning the specification of the interface agents, we have identified six types of interface agent responsible for direct interaction with the human regulator.These agents are represented in the form of interactive windows. The regulator can interact with these agents via the various functions possible in the windows, for example: the buttons, the edition zones, the pictures, etc. These agents are: The State of traffic agent, The State of the line agent, The Station agent, The Vehicle agent, The Message agent, The Overall view agent. It should be remembered that the aim of the IAS is to enable the regulator to visualise, edit, create and transmit information intended for the passengers in the stations and/or vehicles. In order to perform his/her task of regulation correctly, the regulator interacts with these different interface agents. For instance, the State of the traffic interface agent gives a synthetic representation of all the delays concerning mobile units travelling on the network. Thus, with the help of the network support system, it ensures the real time surveillance of vehicle delays on the network supervised. The State of the line interface agent is made up of graphic elements such as stations, route sections, vehicles, $\breve{E}$ ( (see figure 3a). A click on a vehicle directly displays the view (window) of the vehicle interface agent which will deal with any further interaction with the regulator (see figure $3 \mathrm{~b}$ ). The principle is the same when the operator click on a station (see figure 3c). Explanations about the other types of interface agents can be found in [14] or [18].

\section{Evaluation}

We are evaluating and validating the global approach; preliminary results are available in [18]. In parallel with technical tests, evaluation with regulators using the different interactive systems in normal 
Integration of Traffic Management and Traveller Information Systems:

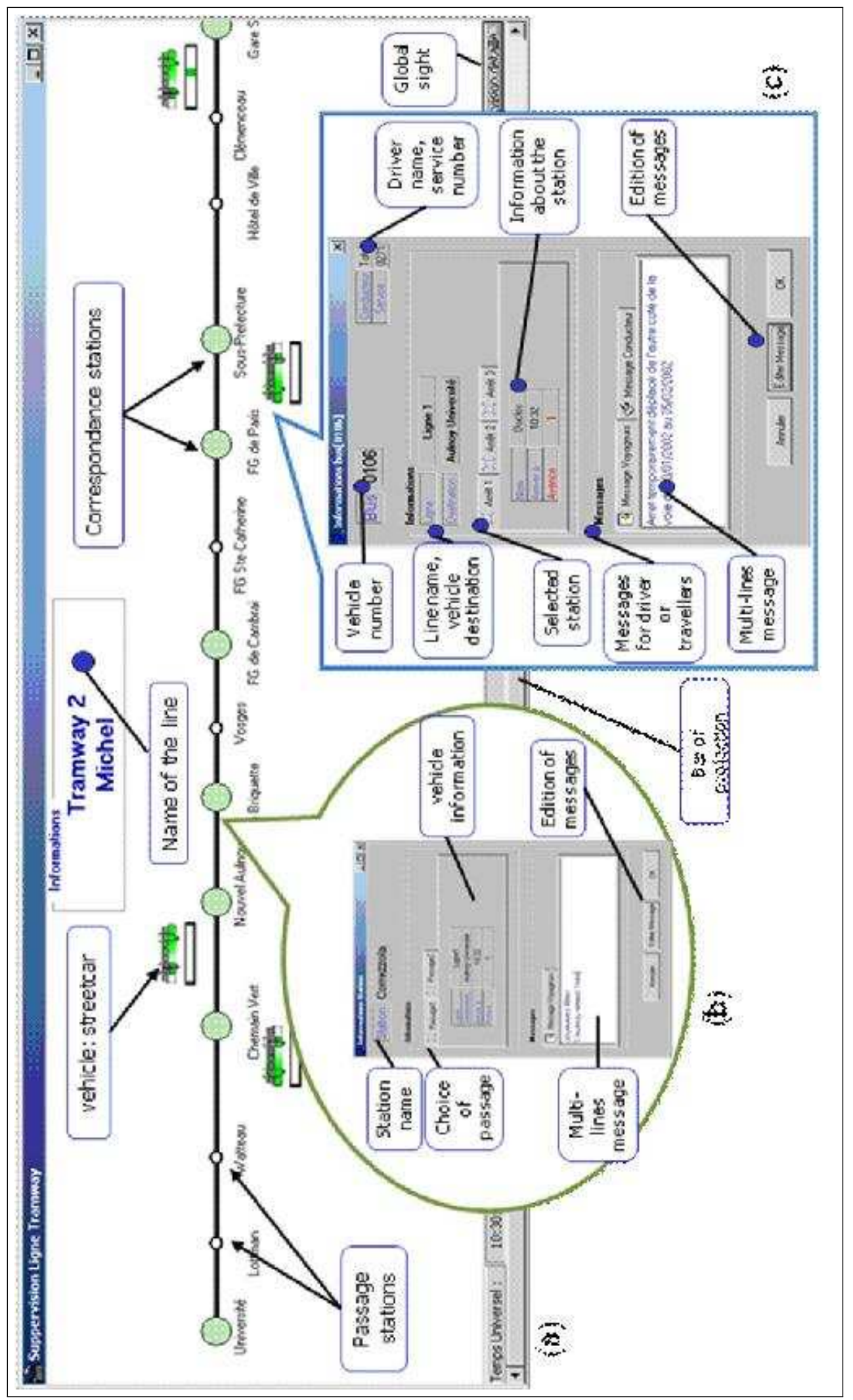

Figure 3: (a) view of the state of the line interface agent, (b) view of a vehicle interface agent, (c) view of a station interface agent 
and abnormal situations are under development. The evaluation of interactive system consists in ensuring that the users are able to carry out their task by using it; it must therefore meet their needs. The methods for evaluating interactive systems are as varied as they are numerous. In $[16,17]$ the emphasis is placed on two global evaluation criteria: utility and usability. Indeed, evaluation consists in verifying and confirming the interactive system, whatever the domain of application. If it meets advanced criteria, it is then accepted and validated. Otherwise it has to be reorganized for a new evaluation.

\subsection{Data Collection using an electronic informer for the evaluation of agent oriented interactive system used in regulation room}

An "electronic informer" is a software tool which ensures the automatic collection, in a real situation, of users' actions and their repercussions on the system. The collection of information is done in a discreet and transparent way for the user, who must not at any time feel hampered by the presence of the informer. Our evaluation method is divided into two closely dependent phases [15]: the first will be dedicated to the acquisition of the data related to the interaction between the user and the agent-based interactive system; the second phase ensures the classification and the analysis of the recovered data.

The parameters to be acquired correspond to the various interactions between the user and the interactive system. They must contribute to the judgement to give on the quality of the system and make it possible to the designers to plan improvements to be brought to it. These parameters allow the identification of the: reactions of the interface further to the actions of the user, reactions of the user further to the actions of the interface, time response of the system, duration of the human tasks, user errors, uses of the help, displayed and not used information, and so on. The acquisition of all these parameters is ensured by the various informer agents. Their tasks will consist in perceiving the actions/reactions of the corresponding interface agent and the user, and to record them. Indeed, we equipped these agents with a rather simple architecture (see Figure 4). This architecture consists of (1) a perception module, ensuring the acquisition of the data provided by the user and the agent presentation, (2) an interpretation

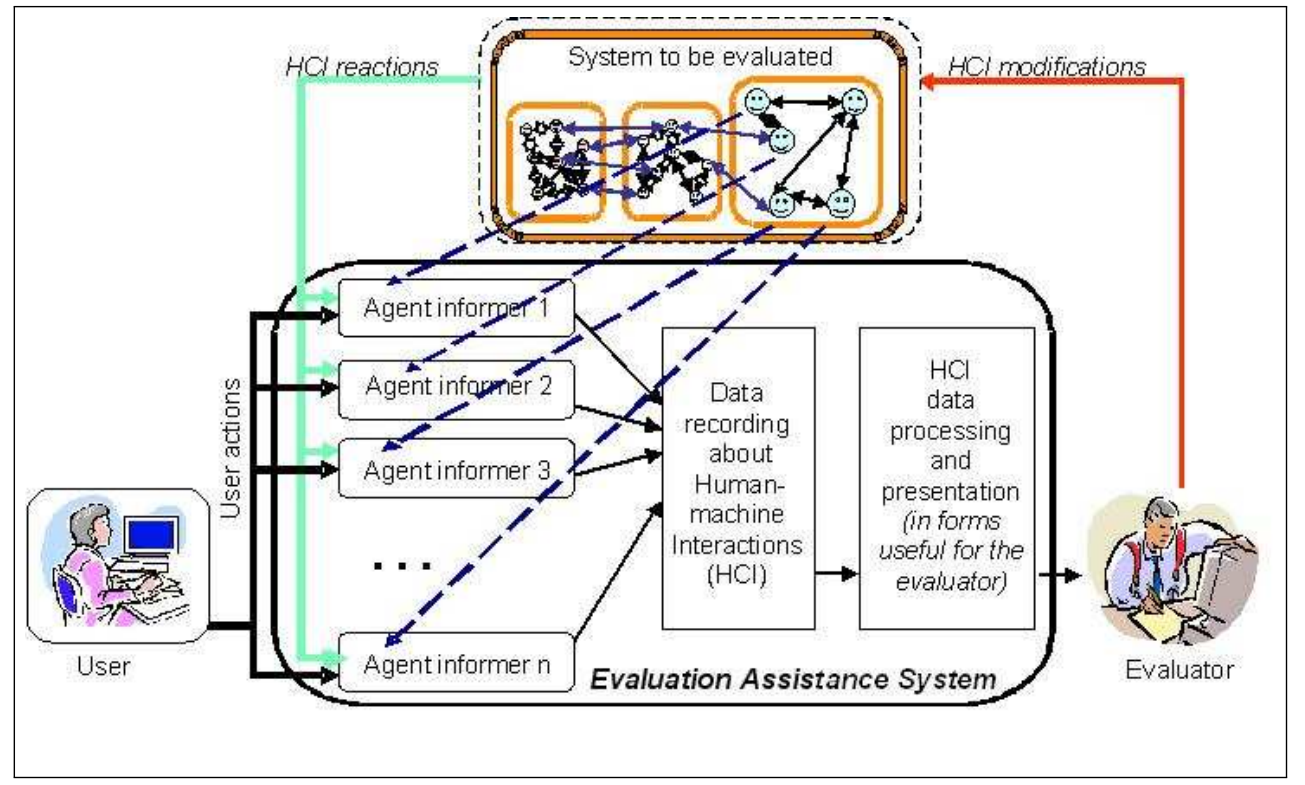

Figure 4: Using the electronic informer for the evaluation of agent oriented interactive system.

and classification module ensuring a sorting of the collected data, and (3) a recording module ensuring the data storage in a database. The acquisition of information via the various tools and techniques of evaluation is not the most difficult task during the evaluation phase of a system. Indeed, the interpretation of the gathered data as their analysis is a more delicate task. 


\subsection{Analysis of the data collected}

The second phase is dedicated to the analysis of the recovered data. In order to facilitate the work of the evaluator, we equipped our electronic informer with an analysis data module. The evaluator can thus consult the various data according to the categories already quoted above. With each appearance of a sight (i.e. the visible part of an interface agent), we engage a stopwatch. It stops at the time of its disappearance or its setting behind plane. The output value represents the duration of the visualization of a sight. We attach importance to the duration of appearance of the sights. Indeed, it can exist a close connection between the duration of consultation of a sight and the strategies implemented by the users. The interaction of the user with the system is carried out via the interface agents; so an agent informer is assigned to each interface agent. In parallel, the integration of a mouse agent and of a Keyboard agent have been done; when collaborating with the other agent informers, the Keyboard and Mouse agents have to allow the recording of different actions/reactions from the interface (interface agents) and from the user. Such principles are more detailed in [18].

\section{Summary and Conclusions}

For economical and ecological reasons, the role of intermodal transportation systems has become increasingly important. It is now crucial to provide the human operators in regulation rooms with systems for making easier their management. A generic model of a traffic regulation support system has been proposed. We are validating the global approach during a project concerning the urban transport network in the town of Valenciennes, France. The article was focused on the decision assistance system and the information assistance system. For the evaluation, an electronic informer for the evaluation of agent oriented interactive system has been realized; its basic principles have been also explained. The proposed approach and the resulting architecture are promising because they are centred on the human activities in regulation room during the different normal and abnormal real situations. Research perspectives concern experimentations in abnormal situations combining different types of dysfunctionnings.

\section{Acknowledgement}

The present research work has been partially supported by the "Ministère de l'Education Nationale, de la Recherche et de la Technologie", the "Région Nord Pas-de-Calais", the FEDER (MIAOU, EUCUE and SART projects). The authors gratefully acknowledge the support of these institutions. The authors thank also the CESA'2006 staff for recommendation.

\section{Bibliography}

[1] M.T. Isaai, N.P. Cassaigne, Predictive and reactive approaches to the train scheduling problem, a knowledge management perspectives, IEEE SMC, Part C, special issue on knowledge management, vol. 31, issue 4, pp 429-437, 2001.

[2] F. Balbo, S. Pinson, Dynamic modeling of a disturbance in a multi-agent system for traffic regulation, Decision Support Systems 41, pp. 131-14, 2005.

[3] F. Cartegnie, H. Ezzedine, A. Peninou, C. Kolski, Agent oriented specification of interactive systems: basic principles and industrial case study, C. Kolski, J. Vanderdonckt (Eds.), Computer-Aided Design of User Interfaces III, Kluwer Academics Publishers, Dortrecht, pp. 381-388, 2002. 
[4] H. Ezzedine, A. Trabelsi, C. Kolski, Modelling of an interactive system with an agent-based architecture using Petri nets, application of the method to the supervision of a transport system, Mathematics and Computers in simulation, vol. 70, Issues 5-6, pp. 358-376, 2006.

[5] B. Fayech Chaar, S. Hammadi, Régulation spatio-temporelle d'un réseau de transport multimodal, e-STA - Sciences et Technologie de l'Automatique, Vol 2, 2005.

[6] P.M. Fitts, J.R. Petterson, Information capacity of discrete motor responses, J. of experimental Psychology, 67, pp. 103-112, 1964.

[7] J.M. Hoc, Supervision et contrôle de processus, la cognition en situation dynamique, Grenoble, PUG, France, 1996.

[8] H. Laichour, S.Maouche, R. Mandiau, Traffic Control Assistance in connection nodes: multi-agent applications in urban transport systems, International Scientific Journal of computing, vol 1, Issue 1, pp. 37-42, 2002.

[9] P. Millot, V. Taborin, A. Kamoun, Two approaches for Man-computer, cooperation in supervisory tasks, 4th IFAC Congress on Analysis Design and Evaluation of HMS, Xian, China, 1988.

[10] Neighbourhood intermodal transfer facilities, L. Land, C. Foreman, Final report, National Center for Urban Transit Research, NCTR university of South Florida, october 2001.

[11] J. Rasmussen, Information processing and human-machine interaction, an approach to cognitive engineering, Elsevier Science Publishing, 1986.

[12] S. Zidi, S. Maouche, S. Hammadi, Nouvelle approche pour la régulation des réseaux de transport multimodal, Geme conférence francophone de Modélisation et SIMulation MOSIM 06, Rabat, Marocco, 2006.

[13] J. Coutaz, L. Nigay, Architecture logicielle des systèmes interactifs, In Kolski C. (Ed.), Analyse et Conception de l'IHM, Vol. 1, pp. 207-246, Paris: Hermès, 2001.

[14] H. Ezzedine, C. Kolski, A. Peninou, Agent oriented design of human-computer interface, application to supervision of an urban transport network, Engineering Applications of Artificial Intelligence, 18, pp. 255-270, 2005.

[15] H. Ezzedine, T. Bonte, C. Kolski, C. Tahon, Intermodal transportation system management: towards integration of traffic management system and users information system, F. Sun, H. Liu (Eds.), IMACS Multiconference on Computational Engineering in Systems Applications (CESA'2006, Beijing, China, oct. 4-6, 2006), Vol. 1, Tsinghua University Press, pp. 972-979, 2006.

[16] J. Nielsen, Usability Engineering, Academic Press, Boston, 1993.

[17] B. Shneiderman, C. Plaisant, Designing the user interface: strategies for effective human-computer interaction, 4th edition, Reading, MA: Addison-Wesley, 2004.

[18] A. Trabelsi, Contribution à l'évaluation des systèmes interactifs orientés agents : application à un poste de supervision du transport urbain (in French), Ph.D. Thesis, University of Valenciennes, France, 2006.

[19] J. Kolodner, Case-Based Reasoning, Morgan Kaufmann, 1993.

[20] M. A. Miller, D. Loukakos, Assessing Opportunities for Intelligent Transportation Systems in California's Passenger Intermodal Operations and Services, California Path research report, November $1,2001$. 
[21] F. G. Filip, Decision support and control for large-scale complex systems, Annual Reviews in Control, Elsevier, Vol. 32, No 1, pp. 61-70, 2008.

H. Ezzedine, T. Bonte, C. Kolski, C. Tahon University of Valenciennes Laboratoire d'Automatique, Mécanique et Informatique industrielles et Humaines LAMIH - UMR CNRS 8530

Le Mont Houy - F-59313 Valenciennes Cédex 09, France E-mail: Houcine.Ezzedine@univ-valenciennes.fr
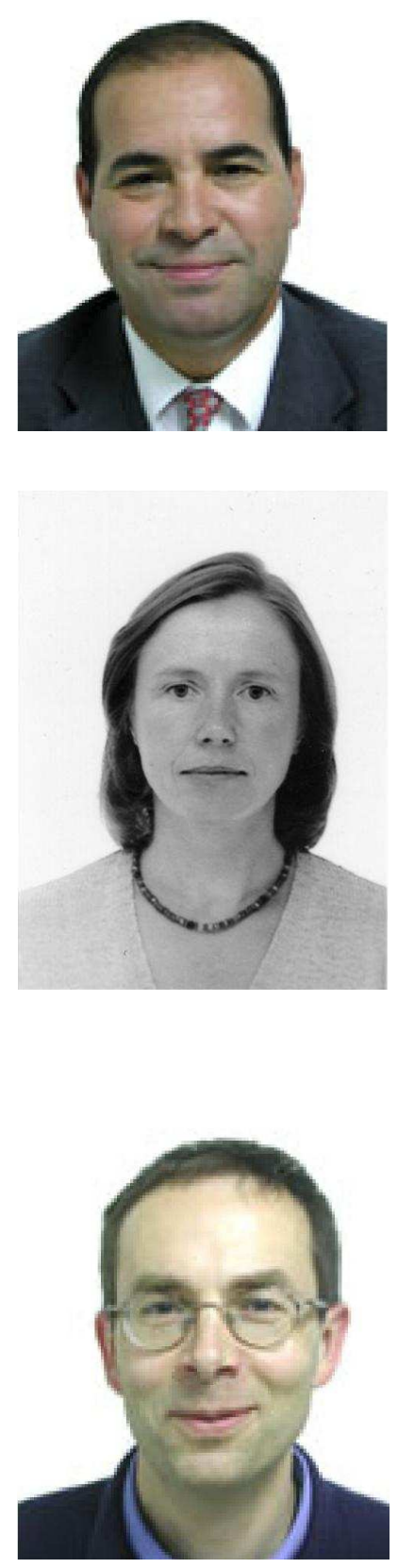

Houcine Ezzedine has obtained his Ph.D in 1985. He is professor in Industrial Computer Science at the University of Valenciennes (France) and member of the "Human-Computer Interaction and Automated Reasoning" research group in the LAMIH. He is involved in several research networks, projects and associations. $\mathrm{He}$ is specialized in human-computer interaction and software engineering for interactive systems.

Therese Bonte has obtained his Ph.D at the University of Valenciennes in 1985 in Simulation for Production Systems. She is researcher in the Production Systems research group of the LAMIH - CNRS laboratory at the University of Valenciennes. She is involved in several research networks and projects. She is specialized in software engineering, simulation and CAD-CAM.

Christophe Kolski has obtained his Ph.D in 1989. He is professor in Computer Science at the University of Valenciennes (France) and head of the "Human-Computer Interaction and Automated Reasoning" research group in the LAMIH. He is involved in several research networks, projects and associations and is a referee for many scientific journals and conferences. He is specialized in human-computer interaction, software engineering for interactive systems and intelligent system design. 


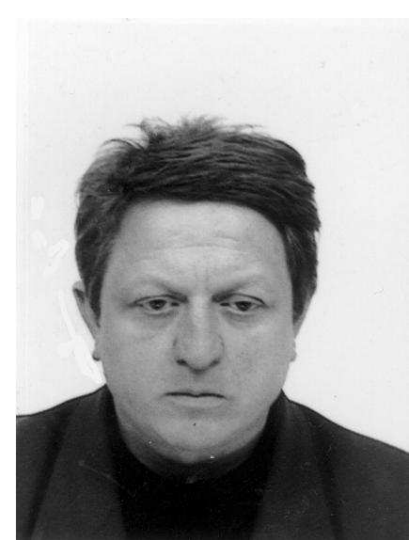

Christian Tahon has an ESTP Engineering School degree. He is qualified in Applied Physics and obtained a Ph.D in Decision Support Systems for Production System Management in 1990 at the University of Valenciennes. He is currently a full professor (France) and head of the Production Systems research group of the LAMIH - CNRS laboratory at the University of Valenciennes. $\mathrm{He}$ is involved in many research and scientific associations and is a referee for scientific journals. His teaching and research interests focus on the areas of decision-support systems, production control systems design, management of transportation systems and services systems. 